\title{
A Low-Jitter Phase-Locked Loop Based on a Charge Pump Using a Current-Bypass Technique
}

\author{
Yongsam Moon
}

\begin{abstract}
A charge-pump circuit using a currentbypass technique, which suppresses charge sharing and reduces the sub-threshold currents, helps to decrease phase-locked loop (PLL) jitter without resorting to a feedback amplifier. The PLL shows no stability issues and no power-up problems, which may occur when a feedback amplifier is used. The PLL is implemented in 0.11- $\mu \mathrm{m}$ CMOS technology to achieve 0.856-ps RMS and 8.75-ps peak-to-peak jitter, which is almost independent of ambient temperature while consuming $4 \mathrm{~mW}$ from a $1.2-\mathrm{V}$ supply.
\end{abstract}

Index Terms - Phase-locked Loop, charge pump, subthreshold current, charge sharing, jitter

\section{INTRODUCTION}

Phase-locked loops (PLLs) have been typically employed in communication integrated-circuits and data interfaces for clock generation and clock recovery. One of the most important PLL characteristics is jitter, which is generated in both transmitter and receiver. Transmitter clock signals are generated by a transmitter-side PLL (TXPLL). Since these clocks switch the serializer, the outgoing data stream inherits the jitter of TXPLLgenerated clock signals. Receiver clock signals sample the incoming data with their own jitter. Thus, the equivalent jitter is the sum of the jitter components in both transmitter and receiver. Because a transceiver operates at higher frequencies and the bit time becomes

Manuscript received Jan. 13, 2014; accepted May. 8, 2014

School of Electrical and Computer Engineering, University of Seoul,

Seoul

E-mail : ysmoon001@uos.ac.kr shorter, the jitter will occupy a greater portion of the bit time and then the equivalent eye-opening will narrow. Therefore, for a lower bit-error rate (BER), jitter must be reduced in both the transmitter and receiver as the frequency increases.

However, in deep submicron CMOS technology (e.g. 0.11- $\mu \mathrm{m}$ CMOS technology used in this design), switch transistors of a charge pump in a PLL exhibit a considerable sub-threshold current when they turn off, thereby injecting leakage current into a loop filter and generating a noisy ripple on a PLL control voltage. Therefore, the PLL jitter increases.

In this paper, a charge pump with reduced subthreshold current is proposed. This paper is organized as follows. Section II discusses the imperfections and solutions of conventional charge-pump circuits. Section III presents the proposed charge-pump circuit for low sub-threshold currents. The experimental results are provided in Section IV. Finally, Section V concludes and summarizes this paper.

\section{Conventional Charge-Pump Circuits}

\section{Improving Matching between UP/DN Currents}

As shown in Fig. 1(a), a conventional PLL consists of a phase frequency detector (PFD), a charge pump (CP), a loop filter (LF), a voltage-controlled oscillator (VCO), and a divider. Several imperfections in the charge-pump circuit lead to high ripple on the PLL control voltage (Vctrl) even in the locked state. Among these nonidealities, the major issue is the mismatch between the up (UP) and down (DN) currents due to channel-length modulation of the current sources. The process, voltage, 


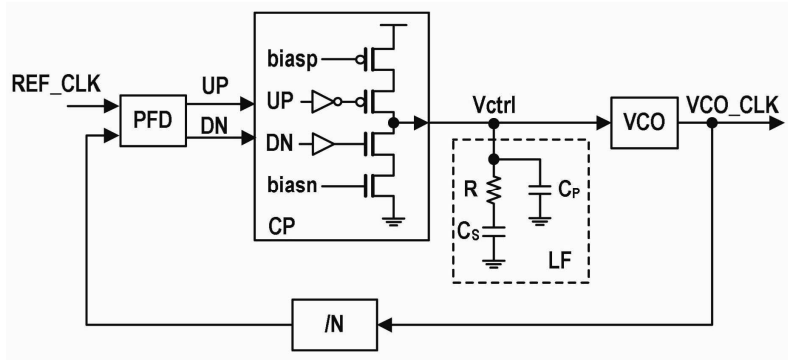

(a)

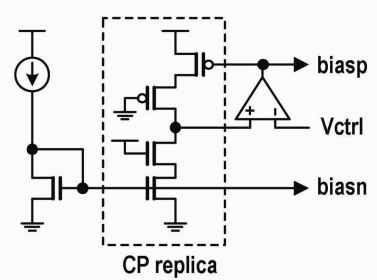

(b)

Fig. 1. (a) Block diagram of a conventional PLL, (b) a chargepump bias circuit.

and temperature (PVT) variations of Vctrl inevitably lead to opposite changes in the drain-source voltages of the UP and DN current sources, thereby incurring a large mismatch.

This issue can be solved by introducing feedback with a single op-amp into the biasing scheme as shown in Fig. 1(b) $[1,2]$. The op-amp senses $V c t r l$ and compares it with the drain voltage of the switch transistors in a CP replica circuit. If this drain voltage is higher than Vctrl, the opamp output (biasp) increases and the UP current of the CP replica circuit decreases slightly, forcing both the UP and DN currents of the charge-pump circuit to be almost equal, regardless of the output voltage.

\section{Sub-threshold Current Issue}

Another problem with a conventional charge pump is sub-threshold current. If UP and DN signals are zero, the switch transistors (MP2 and MN2) turn off and the $\mathrm{UP} / \mathrm{DN}$ currents are expected to be zero. However, in deep submicron technology, considerable sub-threshold currents ( $I_{\text {up.sub }}$ and $I_{\text {dn.sub }}$ ) exist as shown in Fig. 2, although the switch transistors are off. The sub-threshold current of MN2 ( $\left.\mathrm{I}_{\mathrm{dn} . \mathrm{sub}}\right)$ is approximated with the following expression:

$$
I_{d n . s u b}=I_{S} e^{\frac{V_{G S}-V_{T H}}{n \cdot k T / q}}\left(1-e^{-\frac{V_{D S}}{k T / q}}\right)\left(1+\lambda V_{D S}\right)
$$

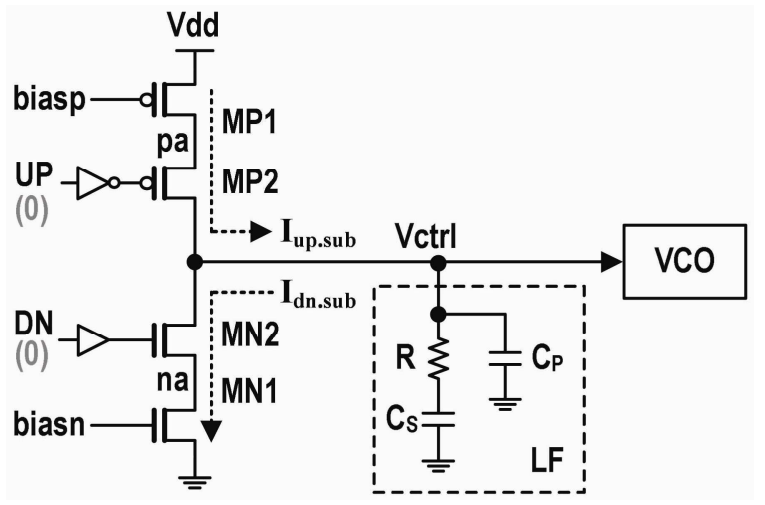

Fig. 2. Sub-threshold currents of a conventional charge-pump circuit.

Here, the equation of the sub-threshold current of MP2 ( $\left.\mathrm{I}_{\text {up.sub}}\right)$ is omitted for simplicity. As shown in Fig. 2, when the switch transistors (MP2 and MN2) are off, MP1 charges node $p a$ to $V d d$ and MN1 discharges node $n a$ to ground. Therefore, the gate-source voltages of the switch transistors are almost zero. The sub-threshold current of MN2 can be expressed as follows (here, $V_{D S}$ of MN2 is V(trl):

$$
I_{d n . s u b @ V_{G S}=0 \& V_{D S}=V c t r l}=I_{S} e^{\frac{-V_{T H}}{n \cdot k T / q}}\left(1-e^{-\frac{V c t r l}{k T / q}}\right)(1+\lambda V c t r l)
$$

As shown in in Fig. 3, simulations using 0.11- $\mu \mathrm{m}$ CMOS technology show that the fast-fast (FF) processcorner model-parameter exhibits relatively larger subthreshold currents compared with the typical-typical (TT) or slow-slow (SS) process-corner model-parameter at the same temperature. The reason is that an FF processcorner transistor has the smaller threshold voltage $\left(\mathrm{V}_{\mathrm{TH}}\right)$. These results coincide with Eq. (2): the sub-threshold currents increase with the decrease in $\mathrm{V}_{\mathrm{TH}}$. As also shown in Fig. 3, if temperature is swept, $\mathrm{V}_{\mathrm{TH}}$ generally decreases with the temperature, allowing the subthreshold currents to increase. These sub-threshold currents are injected into a loop filter and generating a noisy ripple on Vctrl. Therefore, the sub-threshold currents should be reduced.

\section{Reducing Sub-threshold Current}

Fig. 4 shows a charge-pump circuit with a unity-gain 


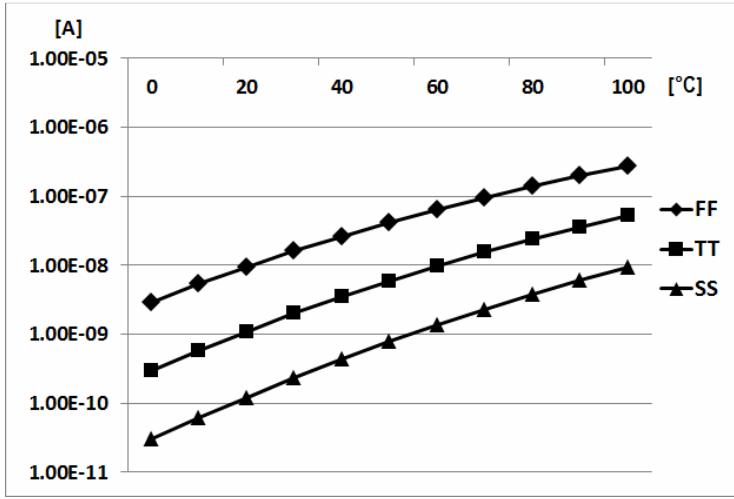

(a)

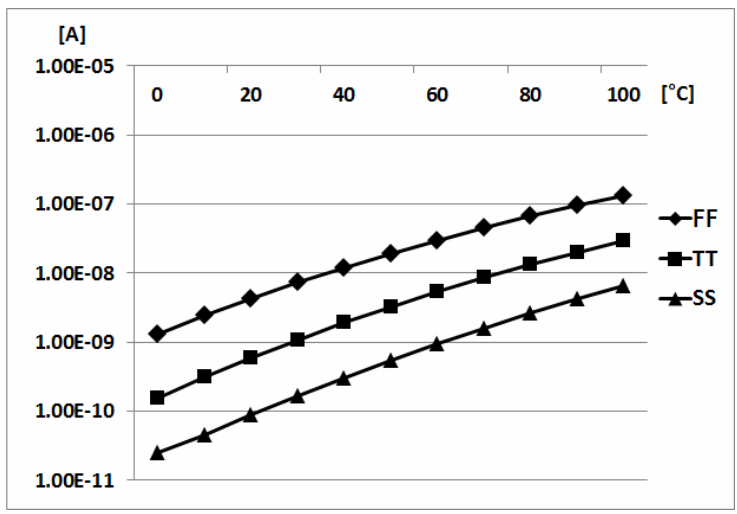

(b)

Fig. 3. (a) UP sub-threshold current ( $\left.\mathrm{I}_{\text {up.sub }}\right)$, (b) DN subthreshold current $\left(\mathrm{I}_{\mathrm{dn} . \mathrm{sub}}\right)$ of a conventional charge-pump circuit.

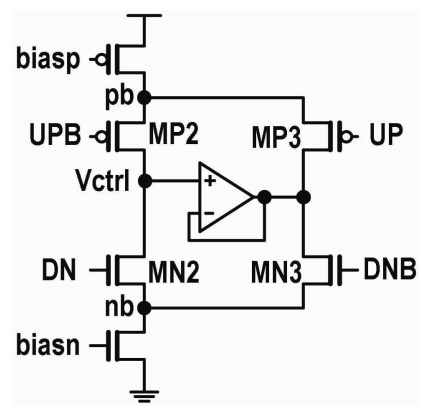

Fig. 4. A charge-pump circuit with a unity-gain amplifier.

amplifier. This circuit was originally designed to suppress charge sharing [3, 4]. As shown in Fig. 4, nodes $p b$ and $n b$ are connected to $V c t r l$ by auxiliary switch transistors (MP3 and MN3) after phase comparison is finished. At the next phase-comparison instant, the main switch transistors (MP2 and MN2) turn on and the nodes $p b$ and $n b$ start with a voltage equal to Vctrl. Therefore, very little charge-sharing can occur between $V c t r l$ and the parasitic capacitances at nodes $p b$ and $n b$.
When the main switch transistors (MP2 and MN2) turn off, the auxiliary switch transistors (MP3 and MN3) turn on and the voltage of nodes $p b$ and $n b$ is set to Vctrl. Therefore, $V_{G S}$ of MN2 is '-Vctrl' and $V_{D S}$ of MN2 is nearly zero. Therefore, the sub-threshold current of MN2 $\left(\mathrm{I}_{\mathrm{dn} . \mathrm{sub}}\right)$ can be expressed with Eq. (3). Compared with Eq. (2), Eq. (3) shows a much reduced sub-threshold current.

$$
I_{d n . s u b @ V_{G S}=-V c t r l \& V_{D S} \approx 0} \approx I_{S} e^{\frac{-V c t r l-V_{T H}}{n \cdot k T / q}}\left(1-e^{-\frac{0}{k T / q}}\right)
$$

In conclusion, this unity-gain amplifier and the auxiliary switch transistors play a role in not only suppressing charge sharing but also reducing subthreshold currents. However, the unity-gain amplifier may bring some operational problems. Generally speaking, Vctrl can have a very wide voltage range according to the PVT variations. So, the unity-gain amplifier needs to work over all the PVT variations and especially over an almost rail-to-rail range of Vctrl. However, the unity-gain amplifier is a feedback circuit so it may have stability issues in a specific PVT corner or at a specific Vctrl. In particular, the unity-gain amplifier may malfunction in a power-up state, resulting in Vctrl being stuck to ground or $V d d$.

\section{Proposed Charge-Pump Circuit using A CURRENT-ByPass TECHNIQUe}

The proposed charge-pump circuit is shown in Fig. 5. Two current bypasses are attached to the original circuit. The UP-current bypass consists of MN4, MN5, MP6, MP1 transistors. The DN-current bypass consists of MP4, MP5, MN6, and MN1 transistors. The transistors in the same row have the same transistor sizes as Eq. (4):

$$
\begin{aligned}
& (W / L)_{M P 1}=(W / L)_{M P 4}, \\
& (W / L)_{M P 2}=(W / L)_{M P 5}=(W / L)_{M P 6}, \\
& (W / L)_{M N 2}=(W / L)_{M N 5}=(W / L)_{M N 6}, \text { and } \\
& (W / L)_{M N 1}=(W / L)_{M N 4} .
\end{aligned}
$$

When UP $=$ ' 0 ' and $\mathrm{DN}=$ ' 0 ', both the current bypasses turn on as shown in Fig. 6(a) and the drain 


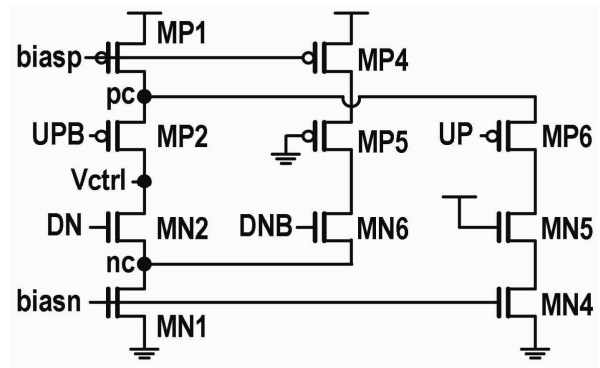

Fig. 5. The proposed charge-pump circuit using a currentbypass technique.

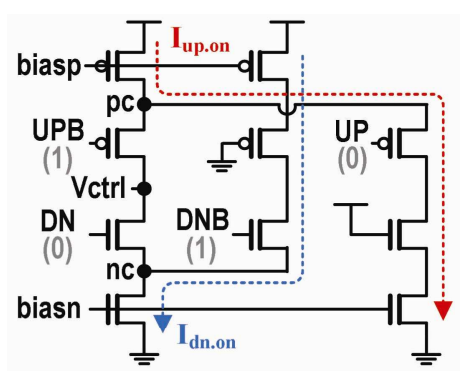

(a)

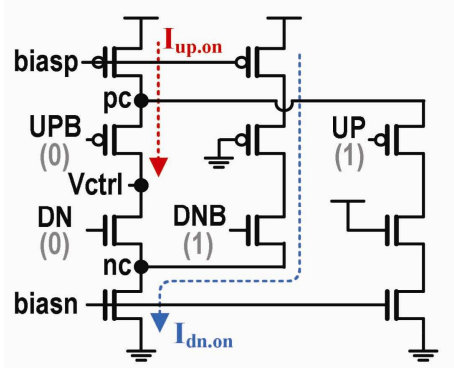

(b)

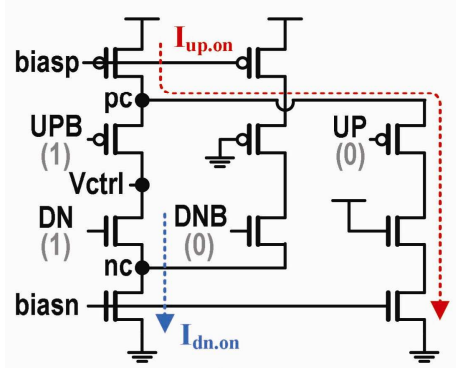

(c)

Fig. 6. The current bypasses of the proposed charge-pump circuit (a) when $\mathrm{UP}=$ ' 0 ' and $\mathrm{DN}=$ ' 0 ', (b) when $\mathrm{UP}=$ ' 1 ' and $\mathrm{DN}=$ ' 0 ', and (c) when $\mathrm{UP}={ }^{\prime} 0$ ' and $\mathrm{DN}=$ ' 1 '.

voltages of the transistors in both the current bypasses are close to Vctrl since MN5, MP6, MP5, and MN6 transistors operate in linear region with small turn-on resistance. So, the voltages of nodes $p c$ and $n c$ stay close to $V c t r l$ as shown in Fig. 7. $V_{G S}$ of MN2 is close to ' - Vctrl' and $\mathrm{V}_{\mathrm{DS}}$ of MN2 is nearly zero. Therefore, the

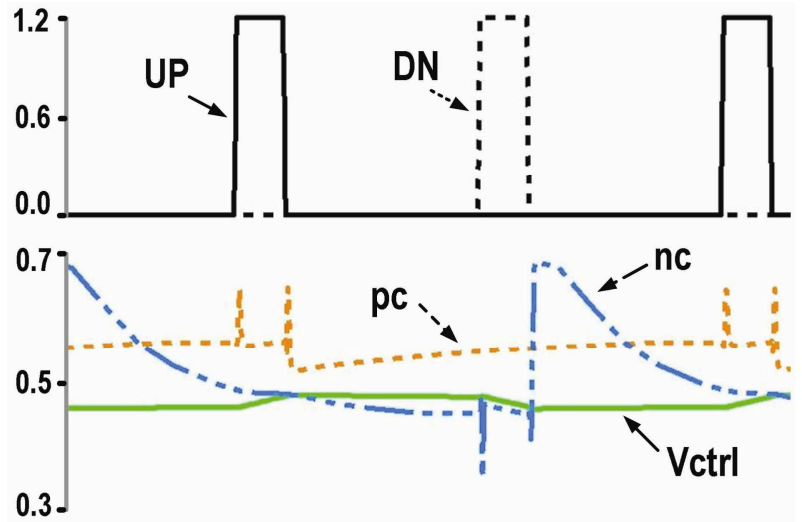

Fig. 7. The simulated voltage waveforms of the proposed charge-pump circuit.

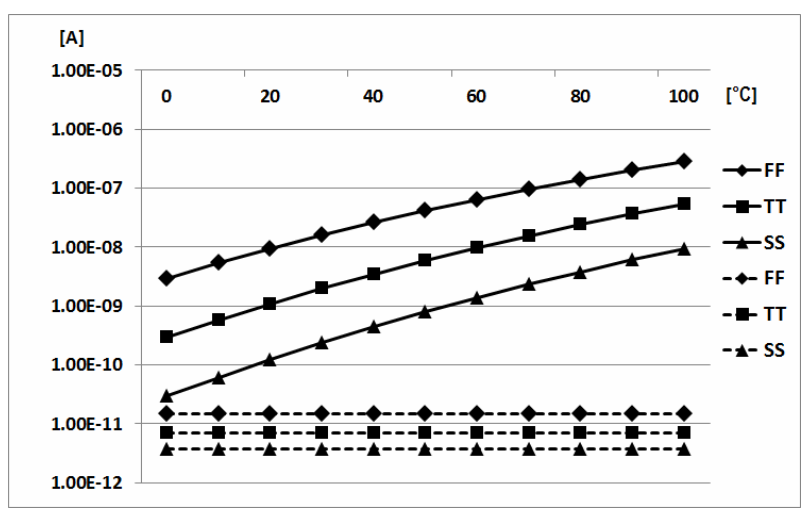

(a)

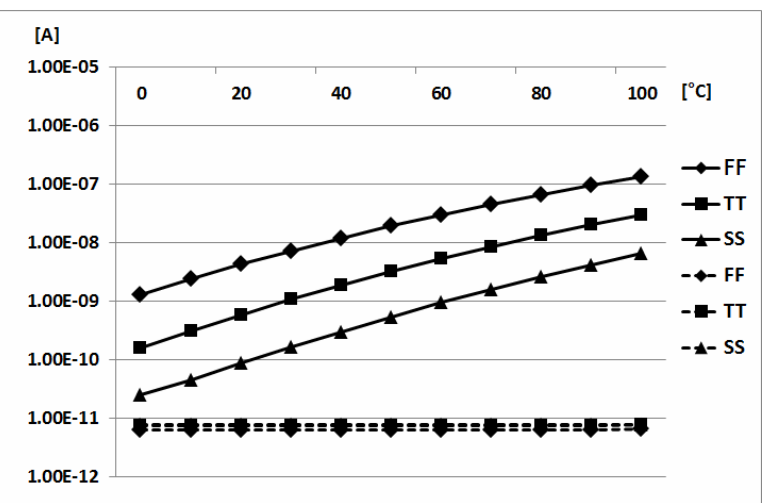

(b)

Fig. 8. (a) UP sub-threshold current ( $\left.\mathrm{I}_{\text {up.sub }}\right)$, (b) DN subthreshold current $\left(\mathrm{I}_{\mathrm{dn} . \mathrm{sub}}\right)$ of the proposed charge-pump circuit. (They are indicated by dotted lines.)

sub-threshold currents of the proposed circuit are reduced to be similar to those of the charge-pump circuit with a unity-gain amplifier in Fig. 4.

When UP becomes ' 1 ' and switch transistor MP2 in the main UP-current path turns on as shown in Fig. 6(b), node $p c$ starts with a voltage close to $V c t r l$ as shown in 


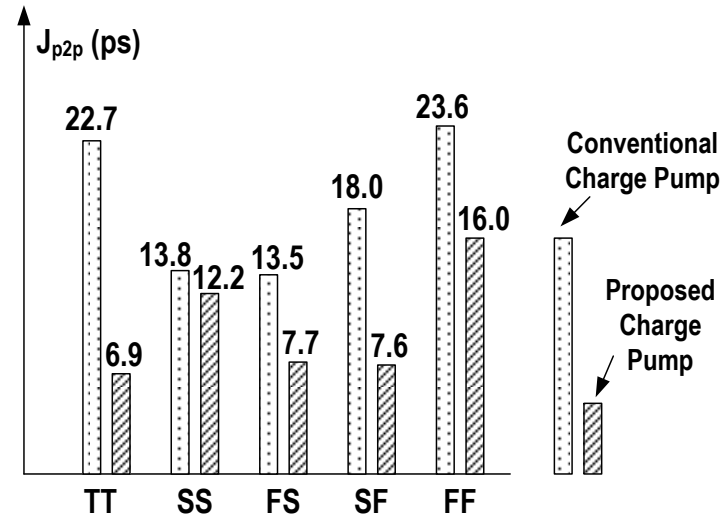

(a)

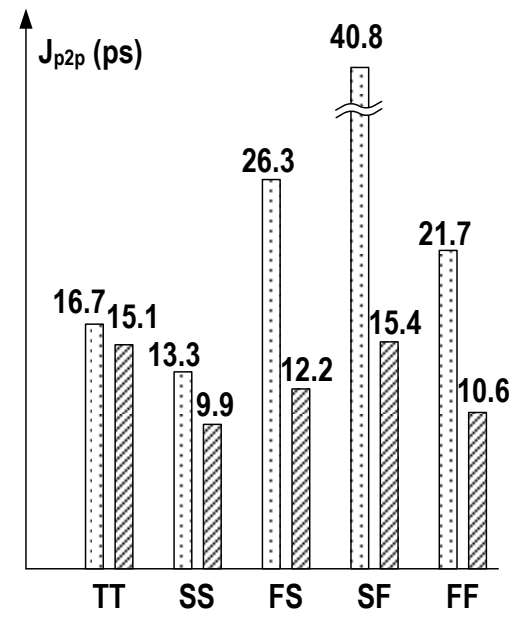

(b)

Fig. 9. Peak-to-peak jitter of a $1.5-\mathrm{GHz}$ VCO clock in various process corners (a) at $0^{\circ} \mathrm{C},\left(\right.$ b) at $100^{\circ} \mathrm{C}$.

Fig. 7. When DN becomes '1' and switch transistor MN2 in the main DN-current path turns on as shown in Fig. 6(c), node $n c$ starts with a voltage close to Vctrl as shown in Fig. 7. Therefore, very little charge-sharing can occur.

According to the simulation results as shown in Fig. 8, the proposed circuit has lower sub-threshold currents (indicated by dotted lines) whereas the original circuit in Fig. 1 has higher sub-threshold currents (indicated by solid lines). The sub-threshold currents of the proposed circuit are around $10 \mathrm{pA}$ and show little dependency on process and temperature variations.

In order to evaluate the effect of the proposed charge pump on jitter, a test circuit for simulation was set up using the PLL configuration shown in Fig. 1 with a 30$\mathrm{MHz}$ reference clock $\left(R E F_{-} C L K\right)$ and a division ratio $(N)$ of 50. Simulations were repeated using the conventional charge pump and the proposed charge

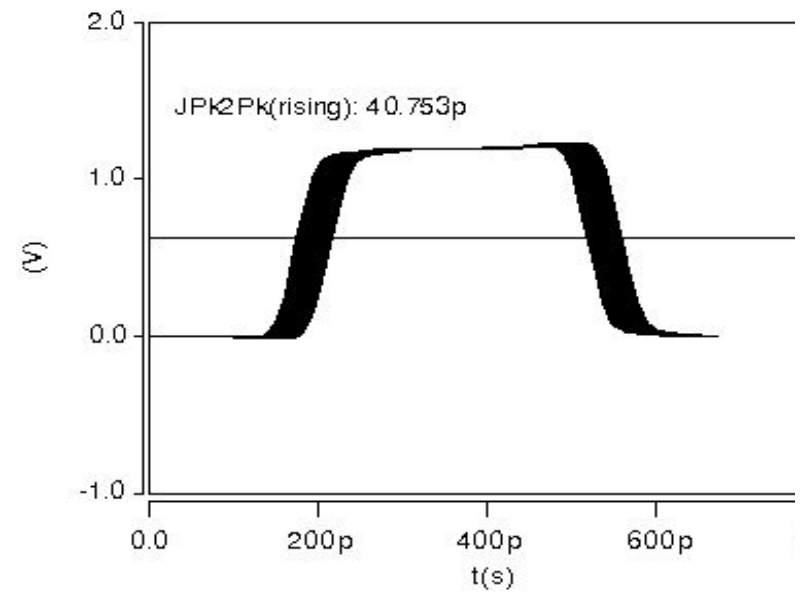

(a)

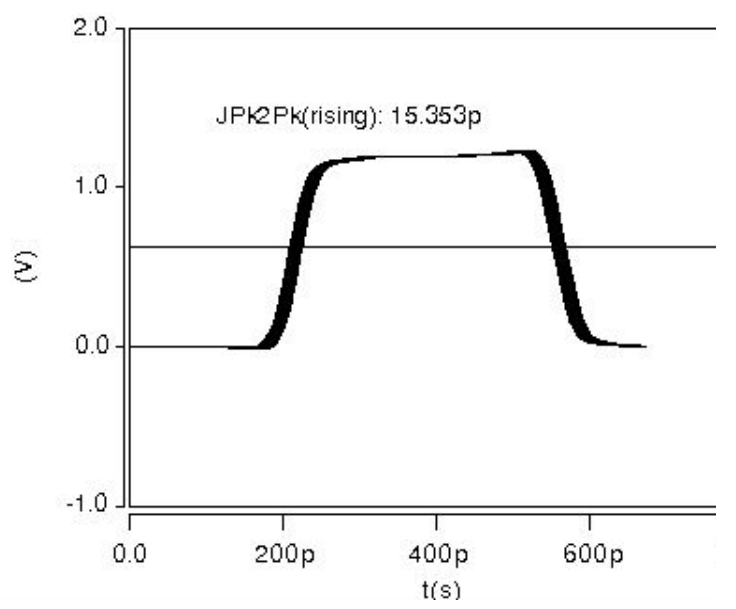

(b)

Fig. 10. Simulated eye diagrams and peak-to-peak jitter of the VCO clock in the SF process corner at $100^{\circ} \mathrm{C}$ using (a) the conventional charge pump and (b) the proposed charge pump.

pump. Fig. 9 shows peak-to-peak jitter of a $1.5-\mathrm{GHz}$ VCO clock (VCO_CLK) in various process corners such as TT, SS, FS (fast NMOS and slow PMOS), SF (slow NMOS and fast PMOS), and FF. Fig. 9(a) shows the jitter at a temperature of $0^{\circ} \mathrm{C}$ and Fig. 9(b) at a temperature of $100^{\circ} \mathrm{C}$.

Simulation results show that the proposed charge pump exhibits relatively lower jitter than the conventional charge pump. Especially in the SF process corner and at $100^{\circ} \mathrm{C}$, the conventional charge pump shows the maximum peakto-peak jitter of 40.8 ps as shown in Fig. 10(a). The reason is that the difference between the UP and DN subthreshold currents ( $I_{\text {up.sub }}$ and $I_{\text {dn.sub }}$ ) is the largest. By contrast, the proposed charge pump shows a reduced amount of 15.4ps as shown in Fig. 10(b). 


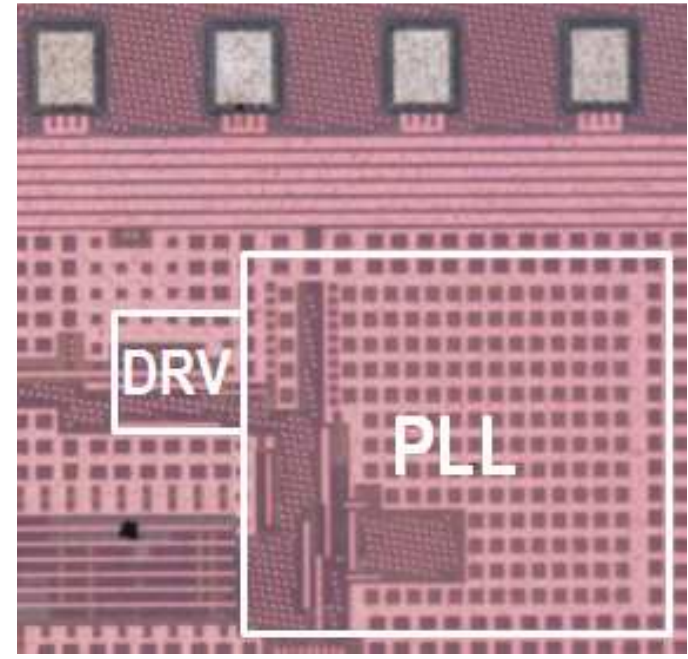

(a)

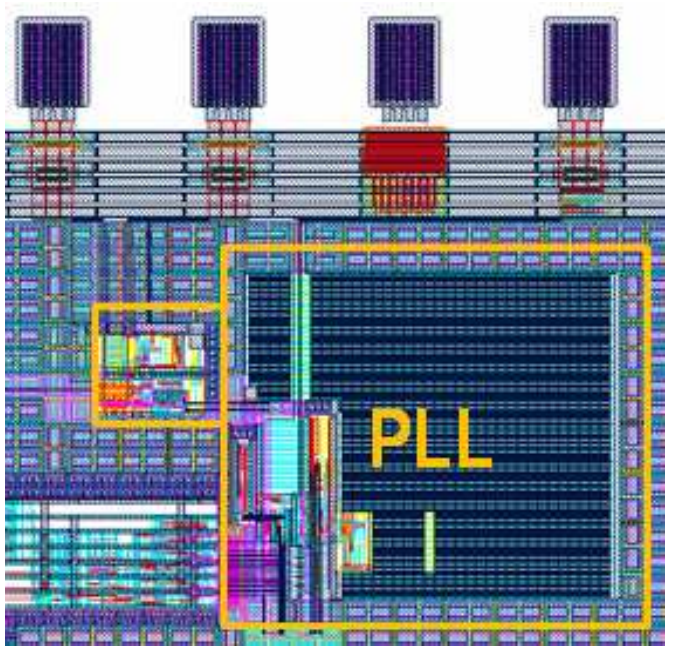

(b)

Fig. 11. (a) Prototype-chip microphotograph, (b) the corresponding layout pattern.

\section{EXPERIMENTAL RESULTS}

Fig. 11(a) shows a microphotograph and Fig. 11(b) shows the corresponding layout pattern of the prototype chip. The prototype chip includes a PLL and an output driver. The output driver receives the $1.5-\mathrm{GHz}$ VCO clock (VCO_CLK) from the PLL and transmits a 3.0Gbps differential clock (1010) pattern through the cables. The prototype chip has been fabricated with $0.11-\mu \mathrm{m} 1$ poly 6-metal logic CMOS technology. Compared with the conventional charge pump, the layout area and current consumption of the proposed charge pump increased by $100 \mu \mathrm{m}^{2}$ and $200 \mu \mathrm{A}$, respectively. The active area of the PLL is $288 \mu \mathrm{m} \times 270 \mu \mathrm{m}$. The power

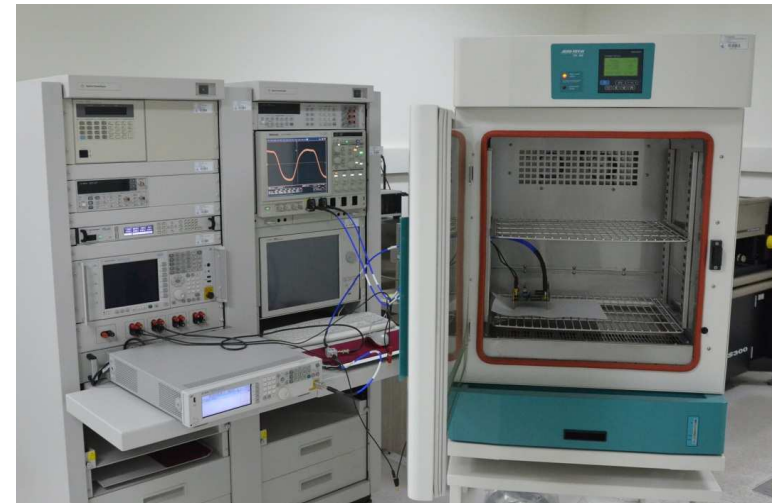

(a)

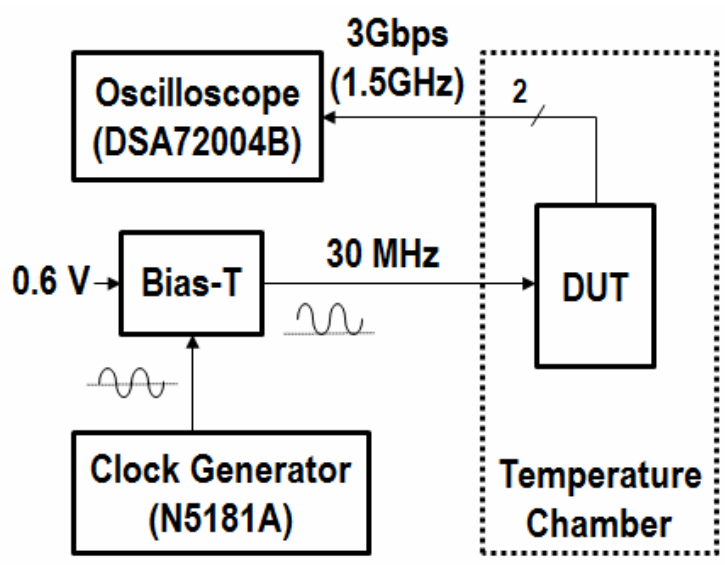

(b)

Fig. 12. (a) Photograph, (b) configuration of the measurement setup.

dissipation is approximately $4 \mathrm{~mW}$ (excluding the output driver) with a $1.2-\mathrm{V}$ supply voltage. The die is directly attached to a test board in a chip-on-board (COB) style.

Fig. 12 shows the measurement setup. An Agilent N5181A generates a $30-\mathrm{MHz}$ clock signal. Through a bias-T circuit, it is used as the reference clock of the PLL in the device under test (DUT). The 3.0-Gbps differential clock signals from the DUT are connected to a Tektronix DSA72004B real-time oscilloscope. The DUT is contained in a temperature chamber, which controls the ambient temperature of the DUT.

With the sweep of the ambient temperature, the eye diagram of the differential driver output, which has a clock (1010) pattern, is measured as shown in Fig. 13. The 3.0-Gbps eye diagram at a chamber temperature of $0^{\circ} \mathrm{C}$ shows 0.747-ps RMS and 5.0-ps peak-to-peak jitter (where the hit count is 10k) as shown in Fig. 13(a). In the eye diagram at $50^{\circ} \mathrm{C}$ shown in Fig. 13(b), the measured 


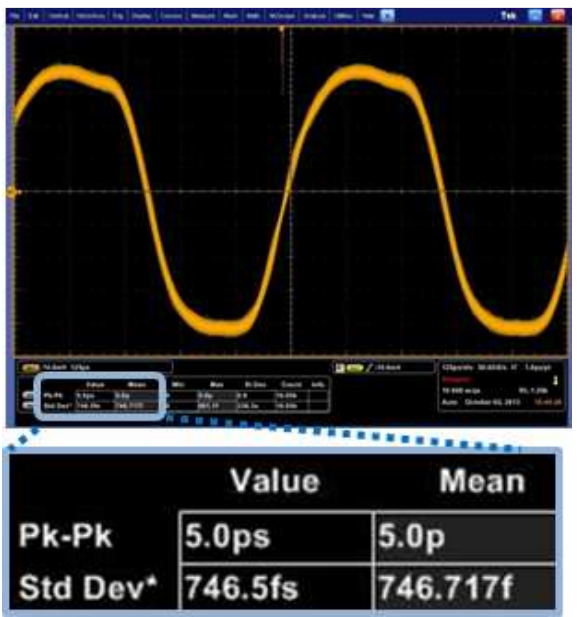

(a)

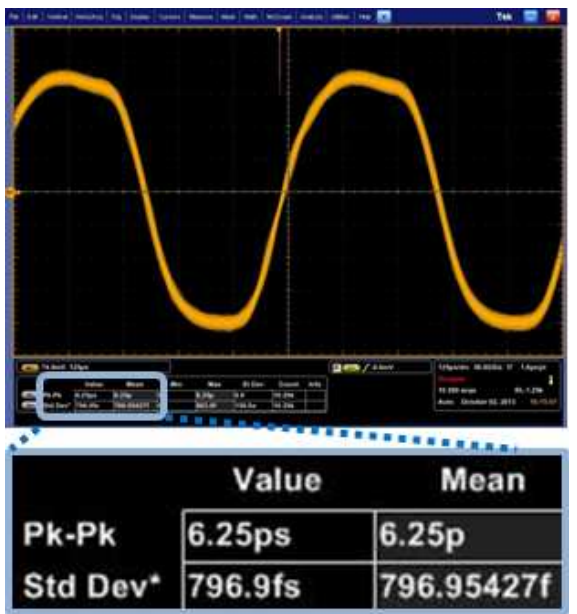

(b)

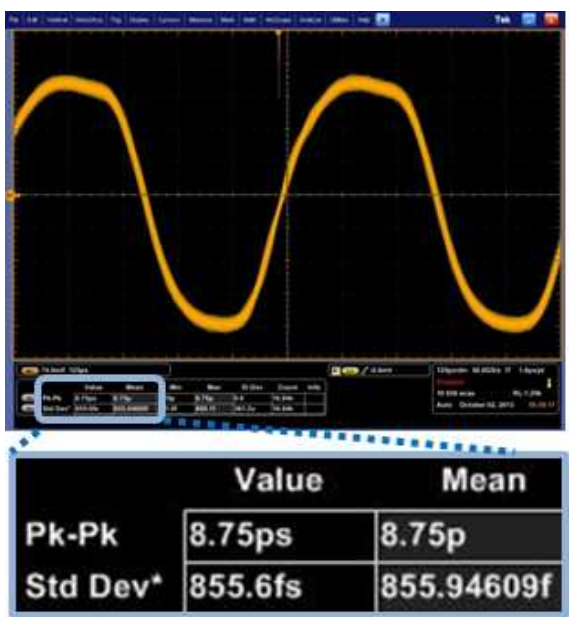

(c)

Fig. 13. Eye diagrams and jitter measurement of the 3.0-Gbps driver output using a clock (1010) pattern at (a) $0^{\circ} \mathrm{C}$, (b) $50^{\circ} \mathrm{C}$, (c) $100^{\circ} \mathrm{C}$.

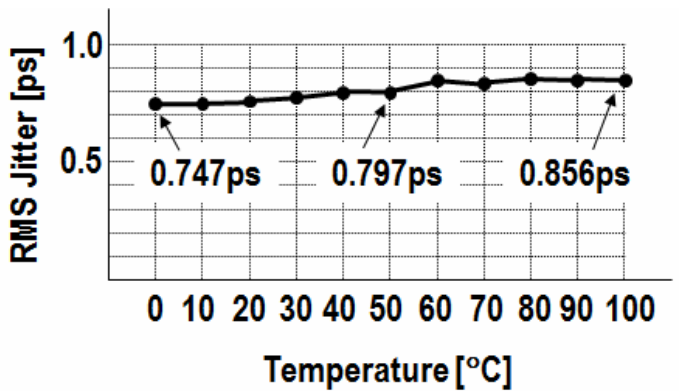

Fig. 14. RMS jitter measurement with the sweep of the chamber temperature.

Table 1. Performance Summary (measurements)

\begin{tabular}{|c|c|}
\hline Process & $0.11 \mu \mathrm{m}$ 1P6M CMOS \\
\hline Circuits & PLL + Driver for test \\
\hline VCO Frequency $\left(\mathrm{f}_{\mathrm{VCO}}\right)$ & $\begin{array}{c}0.82-2.6 \mathrm{GHz} @ 1.2 \mathrm{~V} \\
\text { (nominally } 1.5 \mathrm{GHz})\end{array}$ \\
\hline VCO Gain $\left(\mathrm{K}_{\mathrm{VCO}}\right)$ & $6.0 \mathrm{GHz} / \mathrm{V} @ 1.5-\mathrm{GHz} \mathrm{f}_{\mathrm{VCO}}$ \\
\hline $\begin{array}{c}\text { Measured Jitter } \\
\left(@ 0-100^{\circ} \mathrm{C}\right)\end{array}$ & $<8.75-\mathrm{ps}$ peak-to-peak \\
\hline Power Consumption $(\mathrm{PLL})$ & $4 \mathrm{~mW} @ 1.5 \mathrm{GHz}, 1.2 \mathrm{~V}$ \\
\hline Active Area (PLL) & $288 \mu \mathrm{m} \times 270 \mu \mathrm{m}$ \\
\hline
\end{tabular}

jitter is 0.797 -ps RMS and 6.25-ps peak-to-peak. In the eye diagram at $100^{\circ} \mathrm{C}$ shown in Fig. $13(\mathrm{c})$, the measured jitter is 0.856 -ps RMS and 8.75-ps peak-to-peak. Fig. 14 shows the RMS jitter measurement with the sweep of the chamber temperature. The RMS jitter barely increases with the temperature. Table 1 summarizes the measured performance of the PLL. The VCO shows the frequency range of $0.82-2.6 \mathrm{GHz}$ with a $1.2-\mathrm{V}$ supply voltage.

The PLL operates without a reset signal or an initialization circuit and exhibited no locking problems during the measurements.

\section{Conclusions}

In this paper, we propose a charge-pump circuit using a current-bypass technique. This charge-pump circuit not only suppresses the charge sharing but also reduces subthreshold currents, allowing the PLL jitter to decrease without resorting to a feedback amplifier. The PLL shows no stability issues and no power-up problems, which can occur when a feedback amplifier is used. The PLL in 0.11- $\mu \mathrm{m}$ CMOS technology achieves 0.856-ps RMS and 8.75-ps peak-to-peak jitter, which is almost independent of ambient temperature while consuming $4 \mathrm{~mW}$ from a 1.2-V supply. 


\section{ACKNOWLEDGMENTS}

This work was supported by the 2013 Research Fund of the University of Seoul. The chip was fabricated with the aid of IDEC.

\section{REFERENCES}

[1] J.-S. Lee et al., "Charge Pump with Perfect Current Matching Characteristics in Phase-Locked Loops," Electronics Letters, vol. 36, pp. 1907-1908, Nov. 2000.

[2] M. Terrovitis et al., "A 3.2 to $4 \mathrm{GHz}, 0.25 \mu \mathrm{m}$ CMOS Frequency Synthesizer for IEEE 802.11a/b/g WLAN," IEEE ISSCC Dig. Tech. Papers, pp. 98-99, Feb. 2004.

[3] M. Johnson and E. Hudson, "A Variable Delay Line PLL for CPU-Coprocessor Synchronization," IEEE J. Solid-State Circuits, vol. 23, pp. 12181223, Oct. 1988.

[4] I. A. Young, J. K. Greason, and K. L. Wong, “A PLL Clock Generator with 5 to $110 \mathrm{MHz}$ of Lock Range for Microprocessors," IEEE J. Solid-State Circuits, vol. 27, pp. 1599-1607, Nov. 1992.

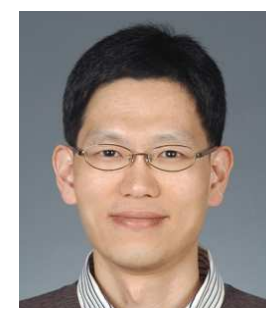

Yongsam Moon received the B.S., M.S., and Ph.D. degrees in electronics engineering from Seoul National University, Seoul, Korea, in 1994, 1996, and 2001, respectively. From 2001 to 2002, he was with InterUniversity Semiconductor Research Center in the same university as a research engineer. In 2002, he joined Silicon Image Inc, Sunnyvale, CA, where he developed various high-speed serial links as a Member of Technical Staff. In 2006, he joined Samsung Electronics, Hwasung, Korea, where he was involved in the design of DRAM products. In 2009, he joined the faculty of the School of Electrical and Computer Engineering, University of Seoul, Seoul, Korea. He is currently an associate professor. His current research interests include clock and data recovery for high-speed communication and high-speed I/O interface circuits. 